\title{
Phaseolus vulgaris extract affects glycometabolic and appetite control in healthy human subjects
}

\author{
Angela Spadafranca ${ }^{1 *}$, Samuele Rinelli ${ }^{1}$, Antonella Riva ${ }^{2}$, Paolo Morazzoni $^{2}$, Paolo Magni ${ }^{3}$, \\ Simona Bertoli ${ }^{1}$ and Alberto Battezzati ${ }^{1}$ \\ ${ }^{1}$ Department of Food, Environmental and Nutritional Sciences (DeFENS), International Center for the Assessment \\ of Nutritional Status (ICANS), Università degli Studi di Milano, Via Celoria 2, 20133 Milan, Italy \\ ${ }^{2}$ Indena S.p.A., Viale Ortles 12, I-20139 Milan, Italy \\ ${ }^{3}$ Dipartimento di Scienze Farmacologiche e Biomolecolari, Università degli Studi di Milano, Via Balzaretti 9, \\ 20133 Milan, Italy \\ (Submitted 16 March 2012 - Final revision received 19 July 2012 - Accepted 20 July 2012 - First published online 9 October 2012)
}

\section{Abstract}

Extracts of Phaseolus vulgaris (beans) are known to reduce glycaemia and food intake in rodents and humans. The present study evaluated the effects of a new, standardised and purified P. vulgaris extract (PVE), when employed as a supplement in a mixed balanced meal (60\% carbohydrates, $25 \%$ lipids and $15 \%$ protein), on glycometabolic and appetite control. To this end, a randomised, double-blind, placebo-controlled study was performed in twelve volunteers. Plasma glucose, insulin, C-peptide, ghrelin and satiety sensation ratings were assessed at baseline and during $3 \mathrm{~h}$ after meal consumption associated with PVE $(100 \mathrm{mg})$ or placebo. Compared with placebo, PVE consumption resulted in lower increments in glucose (+15.4 (sem 5.4) v. 26.1 (sem 7.3)\%, $P=0 \cdot 04$ at 30 min), insulin (+981 (sem 115) $v .1325$ (sem 240) \%, $P=0.04$ between 45 and $120 \mathrm{~min}$ ) and C-peptide ( +350 (sem 27) $v .439$ (sem 30) \%, $P=0.04$ between 30 and 90 min). In the first $2 \mathrm{~h}$, plasma ghrelin decreased similarly in both groups but did not rebound as in placebo thereafter $(P=0 \cdot 04)$. Correspondingly, satiety sensation in the third hour was significantly reduced in the placebo but not in the PVE condition. PVE induced a lower desire to eat than placebo $(P=0.02)$ over the $3 \mathrm{~h}$. In conclusion, PVE supplementation reduced postprandial glucose, insulin and C-peptide excursions, suppressed ghrelin secretion and affected satiety sensations, inducing a lower desire to eat. These results support that further studies are needed to prove the concept of employing PVE as a supplement in mixed balanced meals in obese, glucose-intolerant and diabetic subjects.

Key words: Phaseolus vulgaris: Supplements: Mixed meals: Glucose metabolism: Satiety

The incidence of type 2 diabetes in Western countries is increasing, also in correlation with the rise in obesity, which is now becoming a pandemic ${ }^{(1)}$. This phenomenon is the result of drastic changes in lifestyle with a chronic disequilibrium between food consumption and energy expenditure ${ }^{(2)}$. Diet is the most important modifiable risk factor and the starting point in the treatment of obesity and type 2 diabetes: the primary line of treatment is based on a balanced diet with a proper distribution of nutrients aimed at lowering dietary consumption of saturated fats and refined carbohydrates $(\mathrm{CHO})$ in favour of whole grains, fruits, vegetables and legumes ${ }^{(3)}$. Plant food, with a lower energy density, a lower glycaemic index, and a higher fibre and water content, induces a smaller glucose excursion after a meal. Furthermore, vegetable bioactive compounds show healthy properties when consumed by humans. Several compounds that have the ability to modulate the digestion and absorption of dietary $\mathrm{CHO}$ and fats in the digestive system have been identified and used in combination with low-saturated fat and low-glycaemic index diets. Among the above-mentioned compounds, $\alpha$-amylase inhibitors, such as the synthetic drug 'acarbose', prevent the action of pancreatic $\alpha$-amylase, which breaks down $\mathrm{CHO}$ into oligosaccharides, avoiding the passage through the brush border, and have been used to control the postprandial blood glucose response in type 2 diabetes ${ }^{(4)}$. Protein fractions of many legumes, in addition to soyabeans, are sources of natural inhibitors of hydrolases, such as proteases, amylases, lipases, glycosidases and phosphatases ${ }^{(5-7)}$. Phaseolus vulgaris seeds have been reported to contain $2-4 \mathrm{~g} / \mathrm{kg}$ of non-fibre products that slow $\mathrm{CHO}$ absorption in the small intestine through enzyme inhibition. The administration of $P$. vulgaris extract (PVE) has been reported to have

Abbreviations: CHO, carbohydrates; MixStMeal, mixed standardised meal; PVE, Phaseolus vulgaris extract.

*Corresponding author: A. Spadafranca, fax +392 50319191, email angela.spadafranca@unimi.it 
dose-dependent pharmacological effects in both obese and lean rats with a reduction in food intake, a consequent decrement in weight gain and an improvement in postprandial plasma glucose ${ }^{(8)}$. This starch blocker activity is attributed to the typical bean lectins $\alpha$-amylase inhibitors ( $\alpha$-AI), phytohaemagglutinin and arcelins, which are synthesised as a defence mechanism against insects by plants. $\alpha$-AI1 is the most widely distributed isoform in the common bean $(9-11 \% \text { of the total seed protein })^{(9)}$, and has been shown to provide $\alpha$-amylase inhibitory activity in humans ${ }^{(10,11)}$. A dose-dependent decrease in duodenal amylase activity and a delay in gastric emptying after ingestion of $P$. vulgaris $\alpha$-AI1 extract, when supplemented in powder or tablet form with starch meals, have been reported ${ }^{(12)}$. A follow-up study on subjects with diabetes mellitus has demonstrated decreased postprandial glucose and insulin responses ${ }^{(13,14)}$. A dietary supplementation $(1500-3000 \mathrm{mg} / \mathrm{d})$ of a water extract of $P$. vulgaris (Phase $2^{\circledR}$ ) has been demonstrated to promote weight loss and to reduce postprandial spikes in blood sugar $^{(15,16)}$.

More recently, another PVE, Beanblock ${ }^{\circledR}$ (Indena S.p.A.), has been developed and employed in studies performed on animal models where a suppression of postprandial glycaemia and an anorexic effect were observed ${ }^{(17)}$. This PVE takes advantage of the synergic action of $\alpha$-amylase inhibitory activity, with beneficial effects on carbohydrate metabolism, and of phytohaemagglutinin, with modulating effects on gut hormone secretion ${ }^{(18)}$. Particularly, phytohaemagglutinin binds the brush-border membrane of the intestine, stimulating cholecystokinin and glucagon-like peptide release and hormones with an anorexic effect and playing a relevant role in satiety control ${ }^{(19)}$. However, satiety may be mediated by other physiological effects and specifically through postprandial ghrelin secretion. Ghrelin is a stomach-secreted orexigenic peptide that acts as a hunger signal; intravenous infusion of ghrelin increases food intake and enhances appetite $^{(20)}$; in addition, plasma ghrelin concentrations rise gradually before a meal and decrease immediately after eating $^{(21,22)}$. No study, to our knowledge, investigated the ghrelin postprandial response to PVE, neither in animal models, nor in human subjects

Therefore, the present study performed in healthy human subjects tested the acute administration of PVE as a supplement in a mixed balanced meal with two aims: (1) to evaluate the effects on glucose postprandial response, measuring glucose, insulin and C-peptide concentrations; (2) to investigate the effects on appetite control, assessing plasma ghrelin in association with satiety sensation ratings evaluated by the visual analogue scale.

\section{Subjects and methods}

\section{Subjects}

The present study was performed at the International Center for the Assessment of Nutritional Status (ICANS), University of Milan (Italy). A total of twelve healthy subjects (six females and six males), 20-26 years of age, with a normal weight

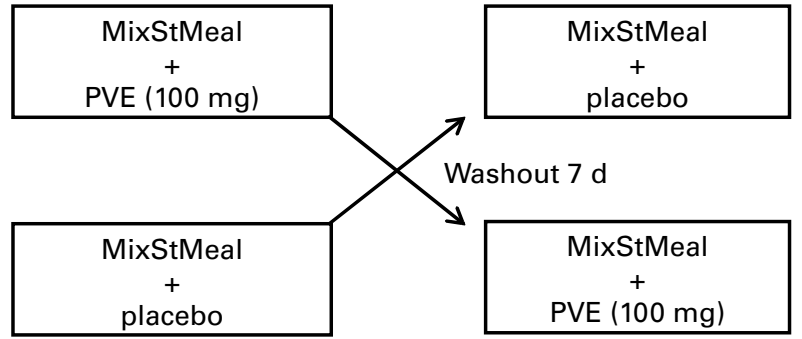

Fig. 1. Experimental design ( $n$ 12). In each experiment, plasma glucose, insulin, C-peptide, ghrelin and subjective satiety sensation ratings (visual analogue scale) were assessed at fasting and after test meal consumption for $3 \mathrm{~h}$. MixStMeal, mixed standardised meal; PVE, Phaseolus vulgaris extract.

(BMI $19 \cdot 7-23 \cdot 5 \mathrm{~kg} / \mathrm{m}^{2}$, body fat $11 \cdot 5-31 \cdot 5 \%$ ) were recruited on a voluntary basis among students of the University of Milan. Exclusion criteria were as follows: overweight or obesity; presence of any disease causing significant impairment of nutritional status (i.e. Crohn's disease, neoplasia, end-stage renal failure, cirrhosis, congestive heart failure and chronic infection); presence of endocrine diseases (i.e. hyper-hypothyroidism and diabetes mellitus); consumption of medications affecting endocrine function within the previous 2 months; recent ( $<1$ month) occurrence of acute illness or injury; elite athletism. The present study was conducted according to the guidelines laid down in the Declaration of Helsinki. Approval was obtained by the Institutional Ethical Committee and an informed consent was signed by all subjects.

\section{Experimental protocol}

The experimental design is described in Fig. 1. In a doubleblind, randomised, cross-over study with a washout period of $7 \mathrm{~d}$, the subjects fasted for at least $12 \mathrm{~h}$, and received on two different days a mixed standardised meal (MixStMeal), consisting of a sandwich of white bread, ham, oil and tomato plus a $100 \mathrm{mg}$ tablet of PVE or a $100 \mathrm{mg}$ tablet of placebo ingested immediately before the meal. The MixStMeal satisfied $40 \%$ of an individual's total energy requirement, estimated by multiplying resting energy expenditure (using the Harris-Benedict equation) ${ }^{(23)}$ by the corresponding physical activity level ${ }^{(24)}$. Of the energy provided by the MixStMeal, $60 \%$ was derived from carbohydrates, $25 \%$ from lipids and $15 \%$ from protein; the content of dietary fibre was $10 \mathrm{~g}$.

The evening before each experiment, we asked subjects to consume a standardised dinner without legumes and consisting of rice or pasta dressed with oil and/or parmesan cheese and/or tomato sauce, meat or fish, vegetables seasoned with olive oil, bread and fresh fruit. The only drink recommended was water.

On the day of the test, the subjects arrived at the laboratory at 08.30 hours: they were seated in a comfortable room and after application of intravenous catheters into an antecubital vein, completed a short questionnaire assessing general well-being and fasting levels of satiety sensations (satiety and desire to eat, evaluated by the following questions: 'How satiated do you feel?' and 'How great is your desire to 
eat?', respectively). Scores were rated on a $100 \mathrm{~mm}$ visual analogue scale and anchored at either end with opposite statements ('not all' and 'very much') ${ }^{(25)}$. The subjects were rescheduled if they had, for example, headache and/or had expressed high ratings for nausea and fullness. Satiety-related sensations were reassessed immediately after test meal consumption and every $30 \mathrm{~min}$ for $3 \mathrm{~h}$. Venous blood samples were obtained at baseline and at 10, 20, 30, 45, 60, 90, 120 and $180 \mathrm{~min}$ after test meal consumption in order to assess plasma glucose, insulin, C-peptide and ghrelin. Blood samples were immediately centrifuged and plasma stored at $-80^{\circ} \mathrm{C}$ until laboratory analyses. Finally, we asked all subjects to fill in a Checklist of Medication Side Effects within $24 \mathrm{~h}$ after the experiments, in order to detect eventual adverse effects of PVE.

PVE used in the present study (Beanblock ${ }^{\circledR} ;$ Indena S.p.A.) is a standardised dry extract containing an $\alpha$-amylase inhibitor and phytohaemagglutinin. In particular, the extract is characterised by a standardised composition of a $\geq 6 \%$ (w/w) tested $\alpha$-amylase inhibitor, with inhibiting activity $\geq 1100 \mathrm{U} / \mathrm{mg}$, and phytohaemagglutinin (haemagglutinating activity $\leq 30$ haemagglutinating units/mg). The manufacturing process is described in detail in Fantini et al. ${ }^{(18)}$.

\section{Laboratory analyses}

We measured circulating levels of glucose and insulin at baseline and every $10 \mathrm{~min}$ in the first half hour and every $30 \mathrm{~min}$ thereafter; C-peptide at baseline and every $30 \mathrm{~min}$, ghrelin at baseline and every $60 \mathrm{~min}$. All parameters were assayed by a commercial kit (Roche Diagnostics) with Cobas Integra 400 Plus and Cobas 411 (Roche Diagnostic), except ghrelin which was assayed by a commercial ELISA kit (Millipore).

\section{Statistical analysis}

Statistical analysis was carried out using the SPSS program version 17. All data are presented as means with their standard errors. Tests for normality confirmed that all dependent variables were normally distributed. Repeated-measures ANOVA followed by the least significant difference test was used to assess the effects of time, treatment and the treatment $\times$ time interaction. Integrated AUC were calculated using the trapezoidal rule. The Student's paired $t$ test was employed to evaluate the difference between AUC values and mean percentage changes. A $P$ value below 0.05 was considered as statistically significant.

\section{Results}

Daily energy intake ranged from 8627 to $12171 \mathrm{~kJ}$ (2062 to $2909 \mathrm{kcal})$, therefore energy consumption by the standardised meal provided from 3443 to $4941 \mathrm{~kJ}$ ( 823 to $1181 \mathrm{kcal}$ ).

Repeated-measures ANOVA showed a significant effect of time on glucose $\left(F_{\text {time }}(8,99)=34 \cdot 7, \quad P=0 \cdot 002\right)$, insulin $\left(F_{\text {time }}(8,99)=29 \cdot 8, \quad P=0 \cdot 003\right)$ and C-peptide $\left(F_{\text {time }}(5,66)\right.$ $=107 \cdot 2, \quad P=0 \cdot 001)$, while no effect of treatment and no treatment $\times$ time interaction were observed (glucose:
$F_{\text {treatment }}(1,22)=1 \cdot 50, P=0 \cdot 25 ; F_{\text {interaction }}(8,99)=0 \cdot 48, P=0 \cdot 82$; insulin: $F_{\text {treatment }}(1,22)=2 \cdot 41, P=0 \cdot 15 ; F_{\text {interaction }}(8,99)=1 \cdot 24$, $P=0.44 ; \quad$ C-peptide: $\quad F_{\text {treatment }}(1,22)=3.62, \quad P=0.08$; $\left.F_{\text {interaction }}(5,66)=0 \cdot 89, P=0.53\right)$.

Fig. 2(a) shows the kinetics of glucose, insulin and C-peptide after PVE or placebo supplemented to the MixStMeal. Fasting plasma glucose, insulin and C-peptide did not differ between the test meal studies.

Postprandial blood glucose concentrations peaked at $10 \mathrm{~min}$ after both tests without significant differences. Plasma glucose concentrations were significantly higher $(P<0.05)$ than baseline until $30 \mathrm{~min}$ after the MixStMeal with placebo, while until 20 min after the MixStMeal with PVE. In addition, at 30 min, we observed for PVE a significant lower percentage of blood glucose increment from basal with respect to placebo $(+15.4(\operatorname{sem} 5 \cdot 4) v .+26 \cdot 1(\operatorname{sem} 7 \cdot 3) \%, P=0.04$; Fig. $2(\mathrm{~b}))$. The $0-180 \mathrm{~min}$ AUC of glucose response did not differ between the PVE and placebo $(P=0 \cdot 6)$.

Postprandial insulin concentrations peaked at $20 \mathrm{~min}$ for PVE and at 30 min for placebo without significant differences. At time periods between 45 and $120 \mathrm{~min}$, insulin increased in percentage less after PVE supplementation than after placebo (+981 (sem 115) v. $1325(\operatorname{sem} 240) \%, P=0 \cdot 04$; Fig. 2(b)). The $0-180$ min AUC of insulin response tended to be lower after PVE supplementation $(P=0 \cdot 1)$.

Postprandial C-peptide concentrations peaked at $30 \mathrm{~min}$ after both tests without significant differences. At time periods between 30 and $90 \mathrm{~min}$, C-peptide increased in percentage less after PVE supplementation than after placebo (+350 (sEm 27) v. 439 (sEm 30)\%, P=0.04; Fig. 2(b)). The 0-180 min AUC of C-peptide response was lower after PVE supplementation $(P=0 \cdot 02)$.

Repeated-measures ANOVA of ghrelin showed a significant effect of time $\left(F_{\text {time }}(3,44)=14 \cdot 5, P=0 \cdot 01\right)$ and the treatment $\times$ time interaction $\left(F_{\text {interaction }}(3,44)=51 \cdot 7, P=0.01\right)$ but no significant effect of treatment $\left(F_{\text {treatment }}(1,22)=0 \cdot 29, P=0.61\right)$. Fig. 3(a) shows the kinetics of ghrelin after PVE or placebo supplemented to the MixStMeal. Fasting plasma ghrelin concentrations were not different between the two test meals. In the first $2 \mathrm{~h}$, plasma ghrelin decreased similarly in both groups (-21 (SEM 3.8)\% PVE $v$. -28 (SEM 4.5)\% placebo, $P=$ NS); however, in the third hour, ghrelin rebounded with placebo, whereas it remained suppressed with PVE $(+7.7(\operatorname{sem} 3.5) v$. -1.4 (SEM 4.1$) \%$ during the interval from 120 to $180 \mathrm{~min}, P=0 \cdot 04$; Fig. $3(\mathrm{~b}))$.

Fig. 3(c) shows the effect of PVE or placebo supplemented to the MixStMeal on satiety and desire to eat expressed as sensation ratings. Repeated-measures ANOVA of satiety and desire to eat showed no significant treatment $x$ time interaction and no effect of treatment. However, a significant effect of time on satiety $(P=0.001)$ and desire to eat $(P=0 \cdot 002)$ was observed.

Baseline visual analogue scale scores $(-5 \mathrm{~min}$ before meal consumption) for the measured variables from the appetite questionnaires were not significantly different between the experiments (data not shown).

Satiety sensations similarly peaked immediately after consumption of both test meals without significant differences, 

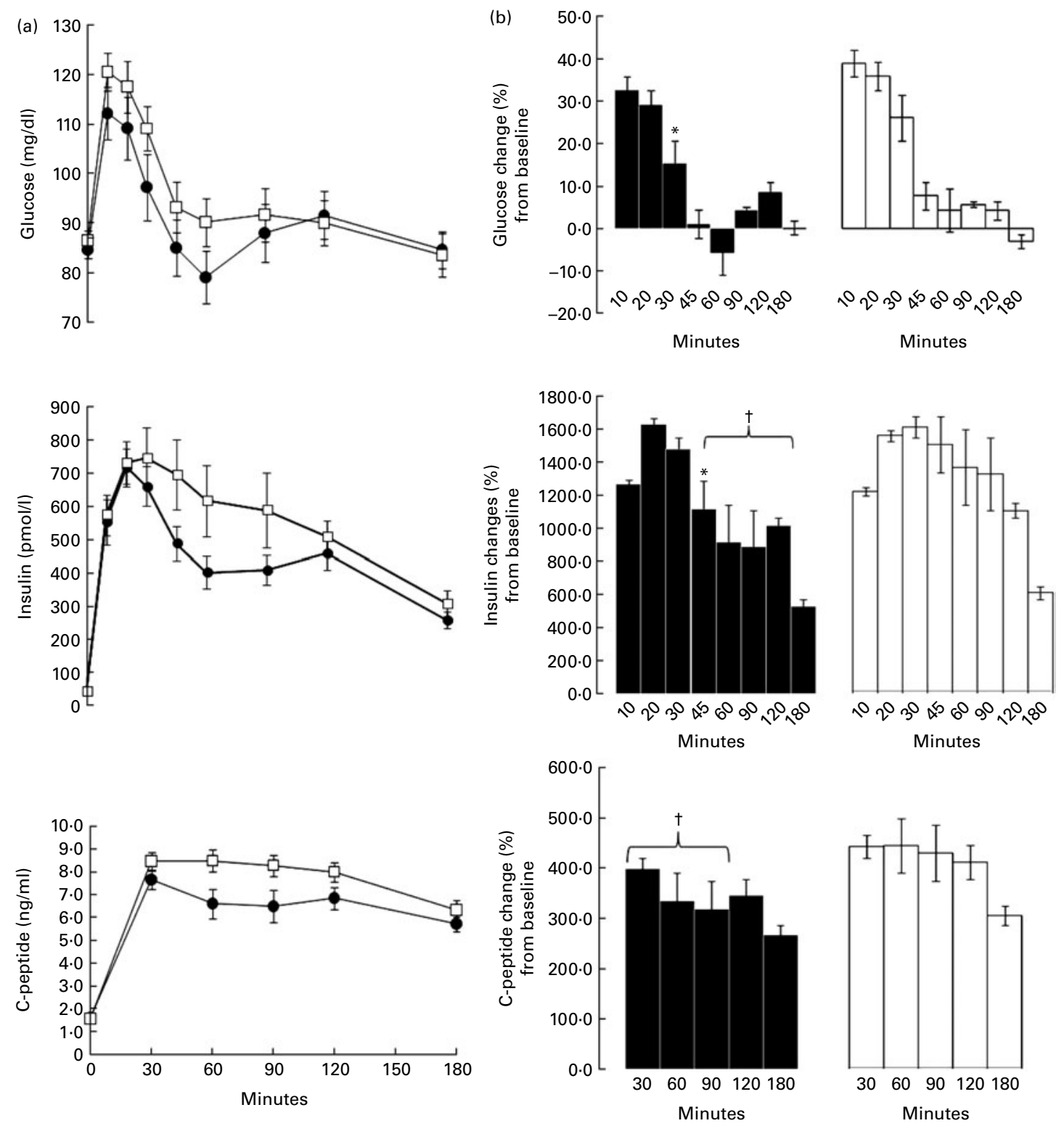

Fig. 2. (a) Fasting and postprandial glucose, insulin and C-peptide responses to the mixed standardised meal (MixStMeal) associated with Phaseolus vulgaris extract (PVE) (•) and placebo $(\square)$. Values are means, with their standard errors represented by vertical bars. There was a significant time effect $(P<0.05$; repeated-measures ANOVA) for glucose, insulin and C-peptide. (b) Glucose, insulin and C-peptide changes (\%) with respect to baseline after the MixStMeal associated with PVE $(\boldsymbol{\square})$ and placebo $(\square)$. * Mean value was significantly different from that of placebo, as percentage increment from basal $(P<0 \cdot 05)$. $†$ Mean value was significantly different from that of placebo, as percentage mean increment from basal during $45-120 \mathrm{~min}(P<0.05)$.

remaining significantly higher than baseline until $180 \mathrm{~min}$ $(P<0.05)$. Despite the similar profiles, satiety significantly reduced from peak later with PVE (after $180 \mathrm{~min}$ ) than with placebo (after $120 \mathrm{~min}$ ).

The desire to eat decreased immediately after both tests, more profoundly with PVE than placebo ( -7.4 (SEM 0.6) $v$. $-6.3($ SEM 0.6$) \mathrm{cm}, P=0.06)$, remaining significantly lower than baseline until $180 \mathrm{~min}(P<0 \cdot 05)$. The $3 \mathrm{~h}$ AUC scores showed that PVE induced lower desire to eat than placebo (PVE: -128 (SEM 38$) \mathrm{cm}^{2} / 180 \mathrm{~min} \quad v$. placebo: -109 (SEM $32 \cdot 4) \mathrm{cm}^{2} / 180 \mathrm{~min}, P=0 \cdot 02$ ).

\section{Checklist of medication side effects}

No significant adverse effects occurred, as no subjects reported any feelings of discomfort within $24 \mathrm{~h}$ after consuming PVE and placebo.

\section{Discussion}

A growing number of studies have indicated that the protein fraction of legumes has beneficial effects on health, improving the postprandial glycaemic response and reducing food intake 

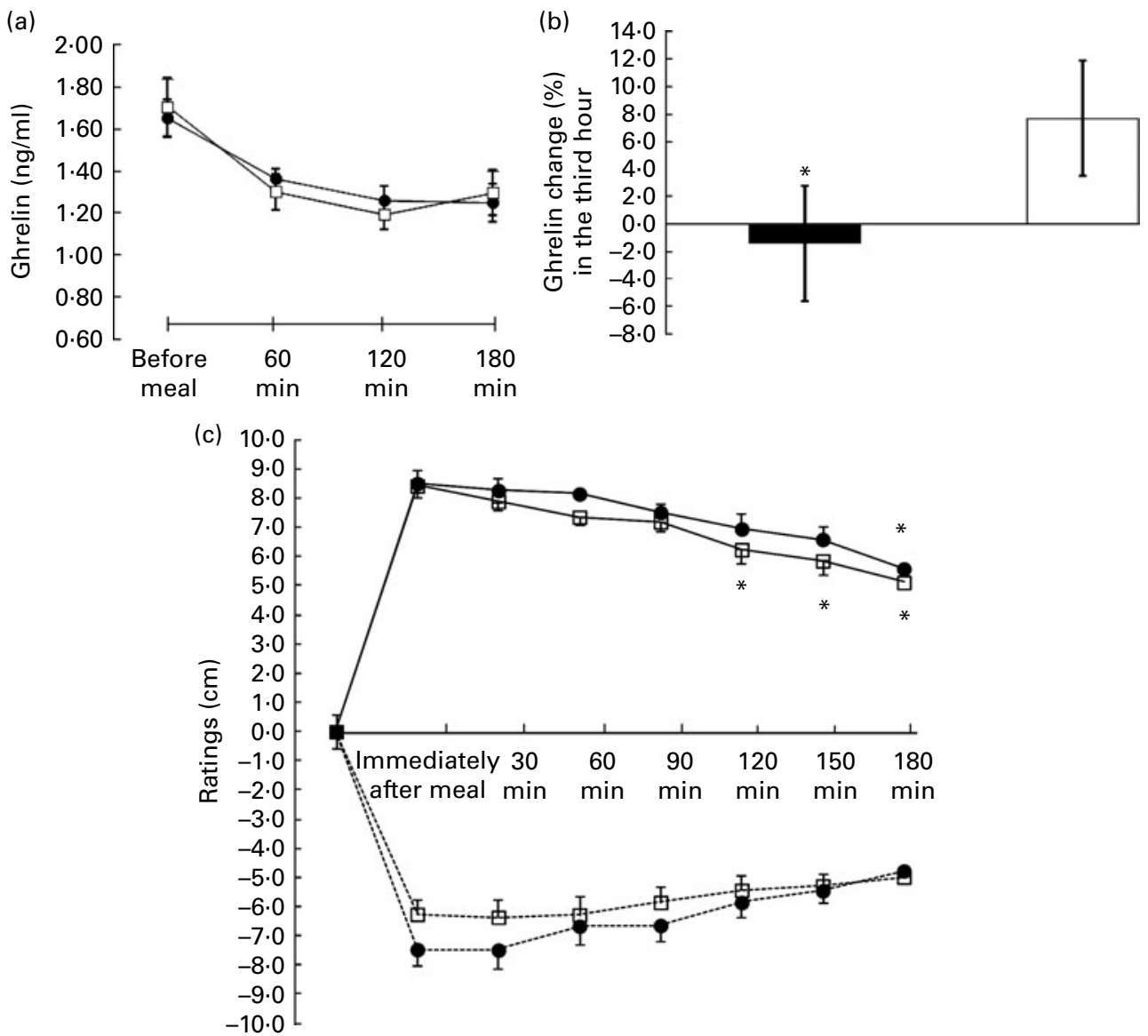

Fig. 3. (a) Fasting and postprandial plasma levels of ghrelin after the mixed standardised meal (MixStMeal) associated with Phaseolus vulgaris extract (PVE) (•) and placebo $(\square)$. Values are means, with their standard errors represented by vertical bars. There was a significant interaction between treatment and time for plasma ghrelin $(P=0.001$; repeated-measures ANOVA). (b) Ghrelin change (\%) between 120 and 180 min after the MixStMeal associated with PVE ( $\mathbf{})$ and placebo ( $\square$ ). Values are means, with their standard errors represented by vertical bars. * Mean value was significantly different from that of placebo $(P=0.04)$. (c) Sensations after the MixStMeal associated with PVE $(\bullet)$ and placebo ( $\square$ ). Satiety $(-)$ and desire to eat (......) over time (subtracted from baseline) registered by the visual analogue scale. Values are means, with their standard errors represented by vertical bars. * Mean value was significantly different from the peak value immediately after the meal $(P<0.05)$. There was a significant time effect for satiety $(P=0.001$; repeated-measures ANOVA $)$ and desire to eat $(P=0.002$; repeated-measures ANOVA).

and weight gain ${ }^{(26,27)}$. The present study is the first to investigate the potential advantages of a new, standardised and purified dry extract of $P$. vulgaris on postprandial glucose metabolism and satiety in human subjects. In a doubleblind, randomised, placebo-controlled trial, we administrated to healthy subjects a balanced mixed meal, representing $40 \%$ of their total energy daily requirement, with or without $100 \mathrm{mg}$ PVE. The amount of the PVE supplement was defined according to recommended safety levels in humans. Chokshi ${ }^{(28)}$ reported that the no observed adverse effect level (NOAEL) in rats for Phase 2, a standardised extract derived from P. vulgaris, is $2500 \mathrm{mg} / \mathrm{kg}$ per $\mathrm{d}$, allowing an acceptable daily intake of $6.0 \mathrm{~g} / \mathrm{d}$ in humans. The PVE employed in the present study showed a NOAEL in rats of $1500 \mathrm{mg} / \mathrm{kg}$ per $\mathrm{d}$ (G Daniela, unpublished results, Università Cattolica del Sacro Cuore, Rome, Italy), allowing an acceptable daily intake of $3.75 \mathrm{~g} / \mathrm{d}$ in humans, assuming a safety factor of 30 as suggested by Chokshi ${ }^{(28)}$. Therefore, $100 \mathrm{mg}$, administered in concomitance with meals to test glycometabolic and appetite control, was a dose providing a broad safety margin because it was widely below the calculated acceptable daily intake.

We showed that PVE supplementation reduced the glucose response to the meal, in association with lower insulin and C-peptide excursions. The present results are consistent with those obtained by Fantini et $a l .{ }^{(18)}$, who demonstrated reduced postprandial glycaemia in Wistar rats treated by intragastric administration of PVE and given unlimited access to a starch-enriched diet and water. In particular, the present study contributes to the mechanistic explanation of this effect because the concomitant reduction in insulin secretion supports the hypothesis that PVE slowed glucose absorption via $\alpha$-amylase inhibition and consequently reducing $\beta$-cell stimulation. This is particularly interesting in the light of recent studies supposing that excessive $\beta$-cell secretory responses lead to hyperinsulinaemia and contribute to obesity, type 2 diabetes and insulin resistance ${ }^{(29)}$. Moreover, it has been reported that postprandial hyperglycaemia and hyperinsulinaemia are associated with the incidence of coronary artery diseases ${ }^{(30,31)}$. Thus, the present results suggest 
that PVE supplementation could be useful and beneficial in diabetic and glucose-intolerant subjects, both with insulin resistance and insulin secretory defect, in order to modulate their postprandial $\mathrm{CHO}$ metabolism and to prevent health complications. These data encourage exploration of this hypothesis in glucose-intolerant subjects.

The second aim of the present study was to investigate the effects of PVE on appetite control, assessing plasma ghrelin levels in association with satiety sensation ratings. We measured ghrelin plasma concentrations before and after mixed meal consumption, and we invited the subjects to register on a visual analogue scale for rating satiety and desire-to-eat sensations. As expected, ghrelin decreased immediately after eating; thereafter it remained suppressed with PVE supplementation while it rebounded with placebo. Moreover, a concomitant reduction in satiety sensation ratings was observed in placebo, but not in PVE. These results led us to hypothesise that PVE could have slowed gastric emptying via inhibition of $\mathrm{CHO}$ hydrolysis, affecting ghrelin secretion and feelings of satiety. Therefore, the modulation of ghrelin secretion could operate through another mechanism, beyond that mediated by cholecystokinin and glucagon-like peptide release ${ }^{(11)}$, contributing to explain the effect of PVE on satiety and control appetite.

In addition, we showed that PVE significantly reduced the desire to eat. Maccioni et al. ${ }^{(32)}$ showed a reducing effect of a PVE on operant self-administration of a chocolate-flavoured beverage in rats: since the content of starch and complex sugars in the chocolate-flavoured beverage was negligible, they excluded a significant role of $\alpha$-amylase inhibitory activity of PVE in the effect observed and hypothesised that additional mechanisms, probably involved in the central regulation of appetite, could be responsible for the satiating effect observed. Since the desire to eat expresses a motivation to eat not solely dependent on a gut-based sensation ${ }^{(33)}$, it is not possible to exclude the intervention of central mechanisms too. Further studies are warranted to better define the mechanisms on the basis of the efficacy of PVE in the control of appetite, with a particular focus on central mechanisms. These findings could be of therapeutic relevance if we consider that often in our clinical practice, obesity and overweight conditions are linked to abnormal craving of very palatable foods.

In conclusion, acute administration of PVE supplemented to healthy subjects reduced postprandial glucose, insulin and C-peptide excursions, suppressed ghrelin secretion and affected satiety sensations, inducing a lower desire to eat. Although these findings are preliminary and limited by the small experimental sample, they encourage further studies to test the effects of PVE supplemented in acute and chronic administration to obese, glucose-intolerant and diabetic subjects in order to evaluate the applicability and potential advantages of this product in these kinds of patients.

\section{Acknowledgements}

The present study was funded by Indena S.p.A. and ICANS (International Center for the Assessment of Nutritional Status), Milan, Italy. The authors thank Massimiliano Ruscica for his technical assistance in ghrelin analysis. The contributions of each author are as follows: S. R., A. B., A. R., P. Morazzoni and S. B. designed the research; A. S., S. R. and P. Magni conducted the research; A. R. and P. Morazzoni provided the PVE; A. S. analysed the data; A. S., S. R., A. B. and $\mathrm{S}$. B. wrote the paper. The authors declare that there are no conflicts of interest.

\section{References}

1. Nolan CJ, Damm P \& Prentki M (2011) Type 2 diabetes across generations: from pathophysiology to prevention and management. Lancet 378, 169-181.

2. Colagiuri S (2010) Diabesity: therapeutic options. Diab Obes Metab 12, 463-473.

3. O'Keef JH, Gheewala NM \& O'Keefe JO (2008) Dietary strategies for improving post-prandial glucose, lipids, inflammation, and cardiovascular health. J Am Coll Cardiol 51, 249-255.

4. Yamagishi S, Matsui T, Ueda S, et al. (2009) Clinical utility of acarbose, an alpha-glucosidase inhibitor in cardiometabolic disorders. Curr Drug Metab 10, 159-163.

5. Duranti M (2006) Grain legume proteins and nutraceutical properties. Fitoterapia 77, 67-82.

6. Belitz HD \& Weder JKP (1990) Protein inhibitors of hydrolases in plants foodstuffs. Food Rev Int 6, 151-211.

7. Ishimoto M, Suzuki K, Iwanaga M, et al. (1995) Variation of seed $\alpha$-amylase inhibitors in the common bean. Theor Appl Genet 90, 425-429.

8. Menezes EW \& Lajolo FM (1987) Inhibition of starch digestion by a black bean $\alpha$-amylase inhibitor, in normal and diabetic rats. Nutr Rep Int 36, 1185-1195.

9. Lee SC, Gepts PL \& Whitaker JR (2002) Protein structures of common bean (Phaseolus vulgaris) $\alpha$-amylase inhibitors. J Agric Food Chem 50, 6618-6627.

10. Chokshi D (2007) Subchronic oral toxicity of a standardized white kidney bean (Phaseolus vulgaris) extract in rats. Food Chem Toxicol 45, 32-40.

11. Carai MAM, Fantini N, Loi B, et al. (2009) Potential efficacy of preparations derived from Phaseolus vulgaris in the control of appetite, energy intake, and carbohydrate metabolism. Diabetes Metab Syn Obes Targ Ther 2, 145-153.

12. Lajolo FM \& Genovese MI (2002) Nutritional significance of lectins and enzyme inhibitors from legumes. J Agric Food Chem 50, 6592-6598.

13. Radberg K, Biernat M, Linderoth A, et al. (2001) Enteral exposure to crude red kidney bean lectin induces maturation of the gut in suckling pigs. J Anim Sci 79, 2669-2678.

14. Preuss HG (2009) Bean amylase inhibitor and other carbohydrate absorption blockers: effects on diabesity and general health bean amylase inhibitor and other carbohydrate. $J \mathrm{Am}$ Coll Nutr 28, 266-276.

15. Wu X, Xu X, Shen J, et al. (2010) Enhanced weight loss from a dietary supplement containing standardized Phaseolus vulgaris extract in overweight men and women. J Appl Res 10, 73-79.

16. Barrett ML \& Udani JK (2011) A proprietary alpha-amylase inhibitor from white bean (Phaseolus vulgaris): a review of clinical studies on weight loss and glycemic control. Nutr J 10, 24 .

17. Carai MAM, Fantini N, Loi B, et al. (2011) Multiples cycles of repeated treatments with Phaseolus vulgaris dry extract reduce food intake and body weight in obese rats. $\mathrm{Br} J$ Nutr 106, 762-768. 
18. Fantini N, Cabras C, Lobina C, et al. (2009) Reducing effect of a Phaseolus vulgaris dry extract on food intake, body weight, and glycemia in rats. J Agric Food Chem 57, 9316-9323.

19. Baintner K, Kiss P, Pfuller U, et al. (2003) Effect of orally and intraperitoneally administered plant lectins on food consumption of rats. Acta Physiol Hung 90, 97-107.

20. Wren AM, Seal LJ, Cohen MA, et al. (2001) Ghrelin enhances appetite and increases food intake in humans. J Clin Endocrinol Metab 86, 5992.

21. Cummings DE, Purnell JQ, Frayo RS, et al. (2001) A preprandial rise in plasma ghrelin levels suggests a role in meal initiation in humans. Diabetes 50, 1714-1719.

22. Tschöp M, Wawarta R, Riepl RL, et al. (2001) Post-prandial decrease of circulating human ghrelin levels. J Endocrinol Invest 24, RC19-RC21.

23. Harris J \& Benedict F (1919) A Biometric Study of Basal Metabolism in Man. Washington, DC: Carnegie Institution. (Publication no. 279).

24. Società Italiana di Nutrizione (SINU) (1996) Livelli di Assunzione Raccomandata di Nutrienti per la popolazione italiana (L.A.R.N.) (Recommended Consumption Levels of Energy and Nutrients for the Italian Population (L.A.R.N.)). http://www.sinu.it/larn/

25. Hetherington M \& Rolls BJ (1987) Methods of investigating human eating behaviour. In Feeding and Drinking, pp. 77-109 [F Toates and N Rowland, editors]. Amsterdam: Elsevier Science Publishers BV.

26. Udani JK, Singh BB, Barrett ML, et al. (2009) Lowering the glycemic index of white bread using a white bean extract. Nutr J 8, 52-56.
27. Tormo MA, Gil-Exojo I, De-Tejada AR, et al. (2004) Hypoglycaemic and anorexigenic activities of an $\alpha$-amylase inhibitor from white kidney beans (Phaseolus vulgaris) in wistar rats. Br J Nutr 92, 785-790.

28. Chokshi D (2007) Subchronic oral toxicity of a standardized white kidney bean (Phaseolus vulgaris) extract in rats. Food and Chem Toxicol 45, 32-40.

29. Corkey BE (2012) Banting lecture 2011 - hyperinsulinemia: cause or consequence? Diabetes 61, 4-13.

30. Cavalot F, Petrelli A, Traversa M, et al. (2006) Postprandial blood glucose is a stronger predictor of cardiovascular events than fasting blood glucose in type 2 diabetes mellitus, particularly in women: lessons from the San Luigi Gonzaga Diabetes Study. J Clin Endocrinol Metab 91, 813-819.

31. Baltali M, Korkmaz ME, Kiziltan HT, et al. (2003) Association between postprandial hyperinsulinemia and coronary artery disease among non-diabetic women: a case control study. Inter J Cardiol 88, 215-221.

32. Maccioni P, Colombo G, Riva A, et al. (2010) Reducing effect of a Phaseolus vulgaris dry extract on operant selfadministration of a chocolate-flavoured beverage in rats. Br J Nutr 104, 624-628.

33. Stubbs RJ, Hughes DA, Johnstone AM, et al. (2000) The use of visual analogue scales to assess motivation to eat in human subjects: a review of their reliability and validity with an evaluation of new hand-held computerized systems for temporal tracking of appetite ratings. BrJ Nutr $\mathbf{8 4}$, $405-415$ 